\title{
Amplification of Cyclin LI is associated with lymph node metastases in head and neck squamous cell carcinoma (HNSCC)
}

\author{
C Sticht ${ }^{1,2}$, C Hofele', C Flechtenmacher ${ }^{3}$, FX Bosch ${ }^{4}$, K Freier ${ }^{1,2}$, P Lichter ${ }^{2}$ and S Joos ${ }^{*, 2}$ \\ 'Klinik für Mund-, Kiefer- und Gesichtschirurgie, Universitätsklinikum Heidelberg, Heidelberg, Germany; ${ }^{2}$ Deutsches Krebsforschungszentrum Abteilung \\ Molekulare Genetik (B060), Im Neuenheimer Feld 280, D-69 20 Heidelberg, Germany; ${ }^{3}$ Pathologisches Institut Universitätsklinikum Heidelberg, \\ Heidelberg, Germany; ${ }^{4}$ Molekularbiologisches Labor der Klinik für Hals-, Nasen-, Ohrenheilkunde, Universitätsklinikum Heidelberg, Heidelberg, Germany
}

Overrepresentation of chromosomal bands 3q25-q29 has been associated with shortened disease-specific survival in head and neck squamous cell carcinoma (HNSCC). To assess the prevalence of copy number gains ( $>4$ signals per cell) and high-level amplifications (> 8 signals per cell) from putative oncogenes in this chromosomal region (CCNL I, SNO, PIK3CA, TP73L), tissue microarray analysis was applied on $280 \mathrm{HNSCCs}$ by fluorescence in situ hybridization. Overall frequency of additional copy numbers was $34.3 \%$ for CCNL I, $31.8 \%$ for SNO, 39.0\% for PIK3CA and 38.3\% for TP73L, respectively. In general, gains were more frequently detected in stage IV compared to stage I-III tumours. Performing multivariate logistic regression analysis, a significant association of CCNLI gains and the presence of lymph node metastases was found, which was independent of anatomical site and T-stage of the primary tumour $(P=0.049)$. Site-specific subgroup analysis further showed that copy number gains of CCNLI and SNO occurred more frequently in oral carcinomas in advanced clinical stages as compared to N0 oral lesions (CCNL I: $P=0.03$; SNO: $P=0.03$ ). Finally, Kaplan-Meier analysis revealed that high-level amplifications of CCNLI correlated with shorter overall survival of the patients. Our results indicate that CCNLI plays a critical role in the loco-regional progression of HNSCC and may serve as an indicator for occult advanced tumour stages.

British Journal of Cancer (2005) 92, 770-774. doi:10.1038/sj.bjc.6602400 www.bjcancer.com

Published online 8 February 2005

(c) 2005 Cancer Research UK

Keywords: head and neck squamous cell carcinoma; oncogenes; Cyclin LI; tissue microarray analysis; FISH

Squamous cell carcinoma of the head and neck (HNSCC) represents the sixth most common human neoplasm. Despite novel combined adjuvant and neoadjuvant therapeutic approaches, the 5-year survival rate did not exceed 55\% during the last decade (Landis et al, 1999). Therapeutic decisions are usually based on clinicopathological parameters like TNM stage and histological grading, which, however, often fail to predict patients' outcome. Therefore, there is a need to better understand HNSCC development on the molecular level. This should lead to an improved stratification between higher- and lower-risk patients, which can be treated in a more selective and individualised manner. Furthermore, understanding molecular pathomechanisms might identify therapeutical interference points.

Copy number gain on chromosome 3 with a minimal overlapping area of bands 3q25-qter is a recurrent molecular alteration in HNSCC (Speicher et al, 1995; Weber et al, 1998). According to Bockmühl et al (2000), this aberration is correlated with a decreased overall survival. The corresponding chromosomal region harbors several potential proto-oncogenes, as for example, TP73L, PIK3CA, SNO and CCNL1, which may contribute to a more aggressive tumour progression. TP73L is a member of the $P 53$ gene family and found overexpressed in various HNSCC cell lines (Hibi

*Correspondence: Dr S Joos; E-mail: s.joos@dkfz.de

Received 27 September 2004; revised 6 December 2004; accepted 17 December 2004; published online 8 February 2005 et al, 2000). PIK3CA, which is involved in multiple cancerrelated functions like cell survival, proliferation and cell migration (Iyer et al, 1999; Guhaniyogi and Brewer, 2001), was considered to play a role in early HNSCC tumorigenesis since genomic amplifications were detected in precancerous oral dysplasias (Redon et al, 2002). Increased expression of PIK3CA, however, failed to predict clinical outcome in a small collection of primary HNSCC (Yang et al, 1998). SNO (SKIrelated novel gene) includes two distinct genes, SNO-A and SNO-N, the latter one being a component of the TGF $\beta / S M A D$ pathway (Stroschein et al, 1999). Amplifications of the gene were previously detected in primary oesophagus squamous cell carcinoma (Imoto et al, 2001). Finally, CCNL1 (also termed Cyclin L1 and Ania-6a) codes for a putative key regulator of pre-mRNA processing and is involved in G0 to G1 transition during the cell cycle (Iyer et al, 1999; Berke et al, 2001). In a recent study, it has been shown to be located on a small region highly amplified in a HNSCC-derived cell line and to be overexpressed in HNSCC primary tumours (Redon et al, 2002).

In the present study, 280 clinically well-defined HNSCCs biopsies mounted on a tissue microarray were analysed for copy number changes of CCNL1, SNO, PIK3CA and TP73L by fluorescence in situ hybridisation (FISH). In this way, we wanted to assess the role of each of these candidate genes with regard to aggressive progression and unfavourable clinical outcome of HNSCC. 


\section{MATERIAL AND METHODS}

\section{Tumour material}

In total, 280 primary, paraffin-embedded HNSCC, 124 oral squamous cell carcinomas (OSCC), 96 pharyngeal squamous cell carcinomas (PSCC) and 60 laryngeal squamous cell carcinomas (LSCC) were obtained from the archives of the Institute of Pathology, University of Heidelberg. For all tumours, histopathological and clinical follow-up data were available. Head and neck squamous cell carcinomas were graded according to the TNM system and the UICC stage. Uvula mucosa tissue from healthy donors was used as reference for FISH experiments. The study was approved by the Medical Ethics Commission, University of Heidelberg.

\section{TMA construction, FISH analysis and statistical evaluation}

Generation of TMAs was performed as previously described (Freier et al, 2003). Briefly, HE-stained sections were cut from each block to define representative tumour regions. Small tissue cylinders with a diameter of $0.6 \mathrm{~mm}$ were taken from selected areas of each donor block using a tissue chip microarrayer (Beecher Instruments, Silver Spring, MD, USA) and transferred to a recipient paraffin block. In all, 280 different tumour specimens and 10 control samples were arrayed. The recipient paraffin block was cut in 5- $\mu \mathrm{m}$ paraffin sections using standard techniques.

For FISH experiments, BAC clones RP11-555M1 (CCNL1), RP11$373 \mathrm{I} 6$ (TP73L), RP11-245C23 (PIK3CA) and RP11-543D10 (SNO), obtained from the Resource Center and Primary Database, Germany (RZPD), were used as probes. Clones were prepared from bacterial cultures using Qiagen-Plasmid-Kit ${ }^{\circledR}$ (Qiagen $\mathrm{GmbH}$, Germany) and labelled by nick translation with cyanine3-dUTP (Perkin-Elmer Life Science, Boston, MA, USA). As internal control, BAC clone RP11-101p149 (chromosome 12q24) was differentially labelled with fluorescein-12-dUTP and co-hybridised.

TMA slides were first deparaffinised in xylene, immersed in $0.2 \mathrm{~N}$ $\mathrm{HCl}$, and incubated in $1 \mathrm{~m}$ sodium thiocyanate solution at $80^{\circ} \mathrm{C}$ for $30 \mathrm{~min}$. Subsequently, they were digested in a protease solution $\left(0.5 \mathrm{mg} \mathrm{ml}^{-1}\right.$ in $\left.0.9 \% \mathrm{NaCl}, \mathrm{pH} 2.0\right)$ for $20 \mathrm{~min}$ at $37^{\circ} \mathrm{C}$. Slides were post-fixed in $10 \%$ buffered formalin for $10 \mathrm{~min}$, dehydrated in ethanol and air-dried. For FISH experiments, $200 \mathrm{ng}$ of labelled probe and control DNA, respectively, were added to a TMA slide in a hybridisation solution containing $50 \%$ deionised formamide, $10 \%$ dextran sulphate, $2 \times$ SSC, $2 \mu \mathrm{g}$ salmon sperm DNA, and $10 \mu \mathrm{g}$ Cot-1 DNA. The TMA slide and probe DNA were denaturated at $75^{\circ} \mathrm{C}$ for $10 \mathrm{~min}$ and hybridised overnight in a humidified chamber at $37^{\circ} \mathrm{C}$. Subsequently, the slides were washed three times in $50 \%$ deionised formamide $/ 2 \times$ SSC at $42^{\circ} \mathrm{C}$ for $10 \mathrm{~min}$ and three times in $2 \times \mathrm{SSC}$ at $42^{\circ} \mathrm{C}$ for $5 \mathrm{~min}$. Interphase nuclei were counterstained with $0.5 \mu \mathrm{g} \mu \mathrm{l}^{-1}$ 4,6-diamidino-2phenylindole (DAPI) in Vectashield mounting medium (Vector Laboratories, Burlingame, CA, USA).

For evaluation of the experiments, hybridisation signals from 25 nonoverlapping interphase cell nuclei of each tumour sample were counted using a fluorescence microscope. A copy number gain was scored, if the average number of signals per nucleus was between 4 and 8 , while a high level amplification was defined as more than 8 signals per cell or if clusters of multiple signals were visible. Fisher's exact test and the $\chi^{2}$ test were performed in order to compare the prevalence of gene copy number gain according to anatomic site, T-stadium, stage and the presence of lymph node metastases. Multivariate logistic regression analysis using the statistical package SPSS (SPSS, Munich, Germany) was applied to all patients with complete clinicopathological data sets. For overall survival analysis, Kaplan-Meier curves of HNSCC subgroups were analysed by $\log$ rank test. To examine the relative impact of oncogene copy number gains on the overall survival of the

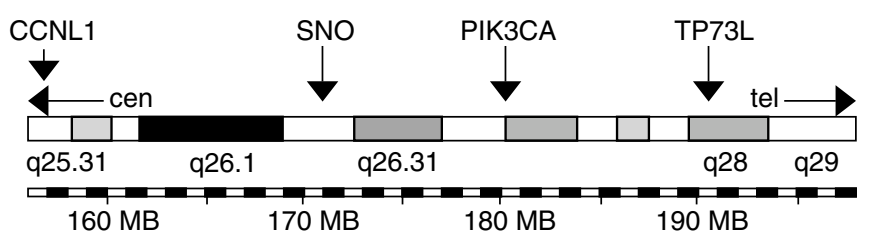

Figure I Localisation of the genes analysed on chromosome 3q25-q29.
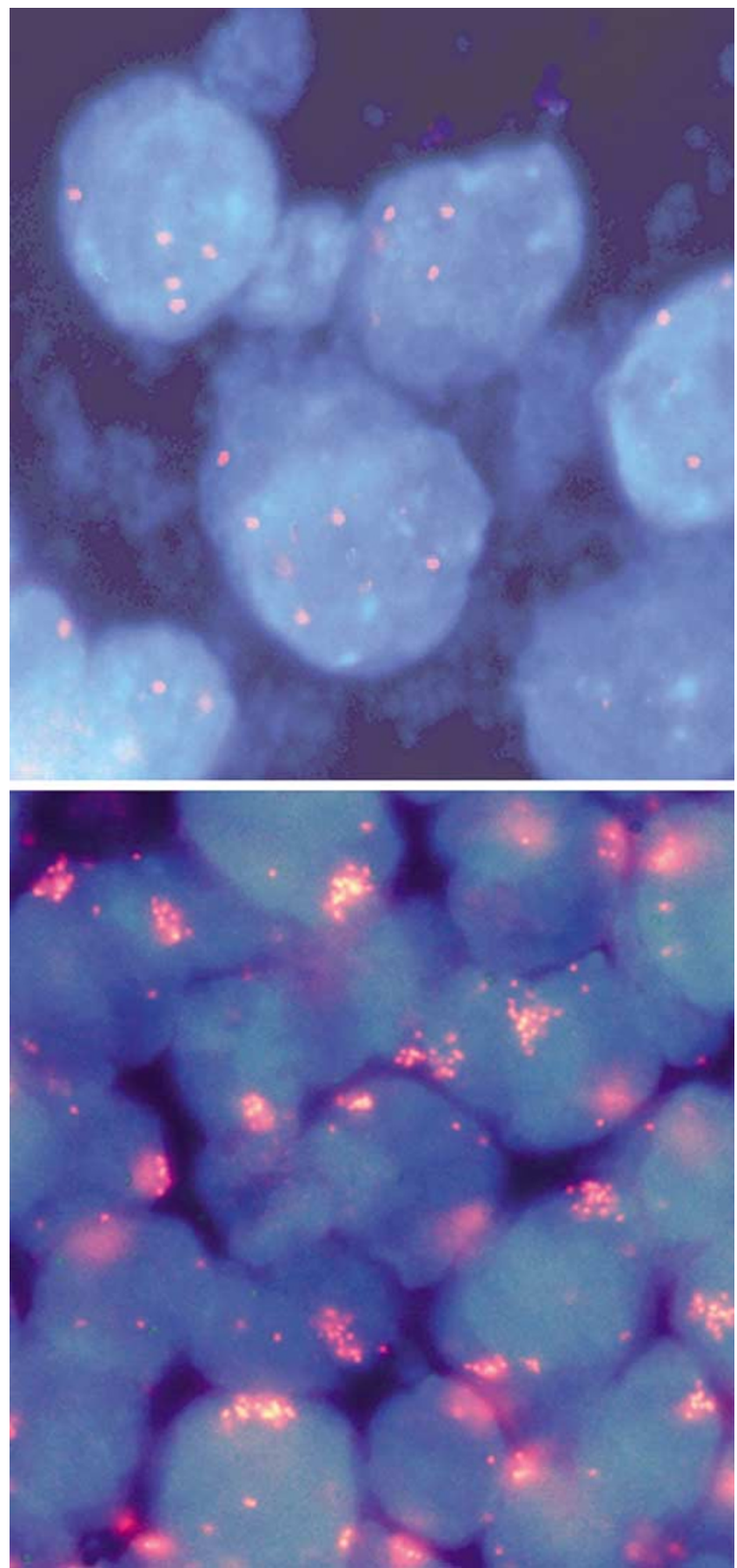

Figure 2 Detection of gene copy number gains by FISH in HNSCCs. Low-level copy number gain of PIK3CA (top) and high-level amplification of CCNLI (bottom). 
patients, a Cox proportional hazards regression model was applied for each oncogene with anatomic site and UICC stage as covariables. $P$-values $\leqslant 0.05$ were considered as statistically significant in all analyses.

\section{RESULTS}

A total of 280 HNSCC tumours from 280 patients were analysed by FISH for copy number changes of proto-oncogenes CCNL1, SNO PIK3CA and TP73L located on chromosomal band 3q25-q29 (Figures 1 and 2). The overall frequency and results from univariate statistical analyses are summarised in Table 1. A significant difference in the prevalence of numerical changes was observed in HNSCC of different anatomical sites, with PSCC exhibiting more copy number gains than LSCC and OSCC (CCNL1 and SNO: $P<0.001$; PIK3CA: $P=0.003$; TP73L: $P=0.007)$. With regard to the clinical stage, the prevalence was significantly higher in stage IV carcinomas as compared to stage I-III carcinomas (CCNL1: $P<0.001$; SNO: $P=0.05 ;$ PIK3CA: $P=0.009$; TP73L: $P=0.03)$. Finally, concerning lymph node metastases, CCNL1 and TP73L showed an increased frequency of gains (CCNL1: $P=0.02$; TP73L: $P=0.05$ ), while for SNO and PIK3CA no such correlation was observed.

To create a multivariate model describing the risk for lymph node metastases, additional clinical parameters were investigated by univariate analysis. A significant association with the prevalence of lymph node metastases was found for higher T-stage (T3/4 vs T1/2; $P \leqslant 0.001$ ), for younger age ( $<60$ years $v s>60$ years; $P=0.005)$ as well as for pharyngeal localisation $v s$ oral cavity $v s$ laryngeal localisation $(P \leqslant 0.001)$. These parameters as well as gain of CCNL1 and TP73L were included in further multivariate analyses. The backward and the forward procedures for variable inclusion/exclusion within the logistic regression model determined pharyngeal localisation $(P \leqslant 0.001)$, higher T-stage $(P \leqslant 0.001)$ and $C C N L 1$ copy number gain $(P=0.049)$ as independent predictors for metastasis formation in HNSCC.

Since HNSCC within the pharynx, lanrynx or oral cavity are different with regard to several clinical parameters (see below), a detailed analysis of the respective genes was performed in OSCC, PSCC and LSCC, separately. In OSCC $(n=85), C C N L 1$ and SNO copy number gains were primarily detected in tumours exhibiting lymph node metastases (CCNL1: $P=0.03$; SNO: $P=0.03$ ). In addition, gains of these genes occurred at higher frequencies in stage IV OSCC as compared to stage I-III OSCC (CCNL1: $P=0.02$;
SNO: $P=0.03$ ) (Figure 3). No such association for any of the oncogenes was found in PSCC $(n=69)$ and LSCC $(n=41)$ (data not shown).

Regarding the clinical outcome, a significant correlation between shortened overall survival and higher T-stage (T3/4 vs T1/2; $P \leqslant 0.001$ ), tumour site (pharyngeal $v s$ laryngeal $v s$ oral cavity; $P=0.008)$ and advanced $\mathrm{N}$-stage $(\mathrm{N} 1-3$ vs N0; $P \leqslant 0.001)$ was found in Kaplan-Meier analysis, whereas no such association for gains of any of the candidate genes was detected $(P>0.05)$. These results were confirmed by multivariate survival analyses including the genes' numerical status, $\mathrm{N}$-stage (N0 vs $\mathrm{N} 1-3$ ), Tstage (T1/2 vs T3/4), tumour site (pharyngeal $v s$ laryngeal $v s$ oral cavity) and age in the Cox proportional hazard models, where only the presence of lymph node metastases $(P \leqslant 0.001)$ and higher Tstage $(P=0.009)$ were significant predictors of shortened overall survival.

Since high-level amplifications might have a stronger impact on tumour formation and biological behaviour than low-level copy number gains (for definition, see Material and Methods), their influence on the clinical course was analysed separately. Although the absolute number of high-level amplifications of the genes analysed was rather low (see Table 1), a correlation with unfavourable clinical course in Kaplan-Meier analysis was found for CCNL1 (log-rank test, $P=0.006$, Figure 4), but not for the remaining genes (all $P$-values $>0.05$ ).

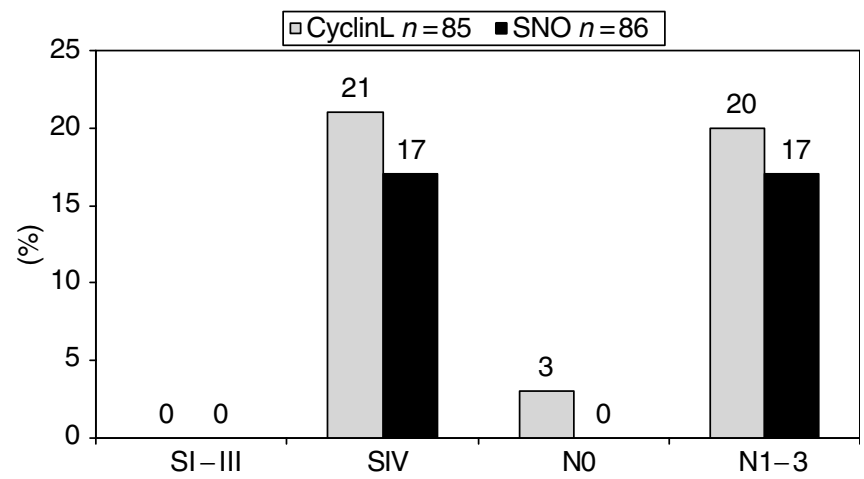

Figure 3 Frequency of CCNLI and SNO copy number gains in OSCC (SI-III and SIV: CCNLI $P=0.02$, SNO $P=0.03 ; \mathrm{NO}$ vs NI-3: CCNLI $P=0.05$, SNO $P=0.05$ )

Table I Frequency of copy number gains of CCNLI, SNO, PIC3CA and TP73L as well as corresponding P-values for univariate subgroup analysis

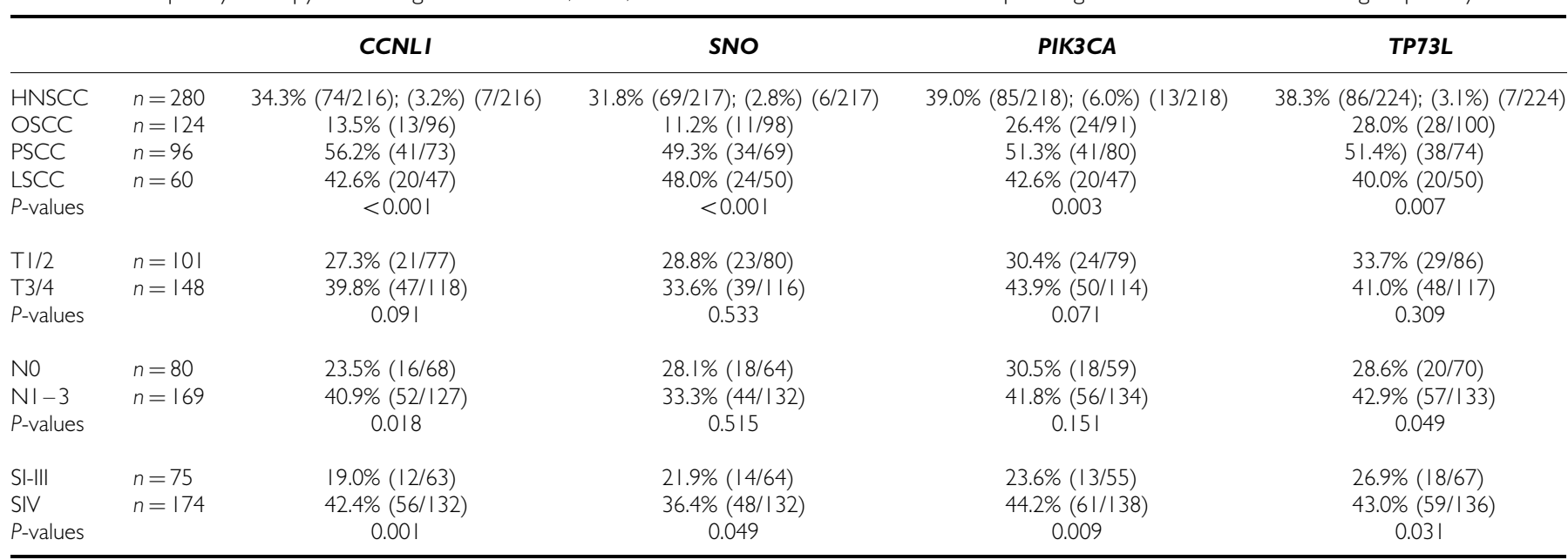

The numbers in parentheses in the first line represent the number of tumours with distinct high-level oncogene amplification in all HNSCC. 
CCNL1

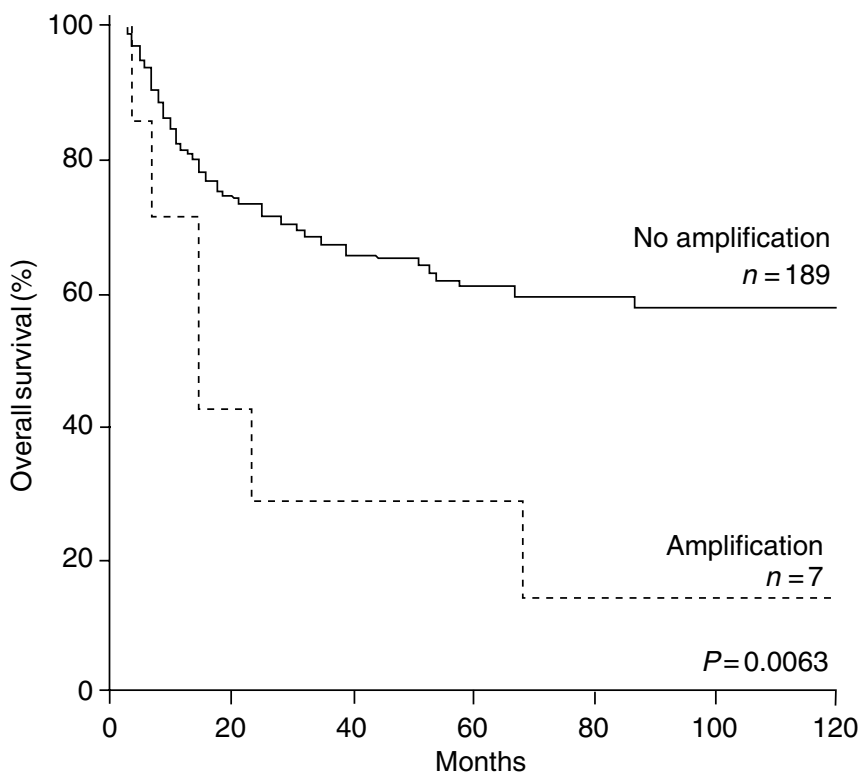

Figure 4 Kaplan-Meier survival analysis of HNSCC with or without CCNLI high-level amplification.

\section{DISCUSSION}

In the present study, the tissue array approach was applied in order to analyse copy number gains of putative oncogenes on a collection of 280 clinically well-documented HNSCC. We found significant associations between clinical parameters and copy number gains of four candidate genes, TP73L, PIK3CA, SNO and CCNL1 located on chromosomal arm 3q. In general, frequencies of copy number gains were between $31.8 \%$ (SNO) and $39.0 \%$ (TP73L). Pharyngeal tumours (PSCC) showed the highest frequency in this respect, and this was significant on the univariate as well as on the multivariate levels (i.e. independent of the tumour size and metastasis status). It is well known that, in contrast to laryngeal and OSCCs, PSCCs are more aggressive, since they exhibit a higher tendency to metastasise and are associated with decreased overall survival (Baatenburg de Jong et al, 1993). A dosage effect of the analysed genes might therefore well contribute to this aggressive phenotype.

One of the major aims of this study was to search for a molecular marker, which is associated with a higher tendency of lymph node metastasis formation. Such a marker should be highly useful during the initial tumour staging and might give additional information concerning the presence of occult metastases. We found CCNL1 to be more frequently increased in copy number in HNSSC with loco-regional metastases, irrespective of anatomic site and T-stage. Such an impact of CCNL1 was also detected in OSCCs when analysed separately from PSCC and LSCC, suggesting that CCNL1 could serve as a molecular indicator for loco-regional metastasis formation in HNSCC. In order to confirm this, however, further prospective studies have to be performed.

CCNL1 was also interesting with respect to overall survival, in that high-level gene amplification ( $>8$ signals/cell) was primarily detected in patients with lower survival rates. It should be noted that high-level amplification of CCNL1 was found to be a rather rare event and therefore the number of cases is still low, not allowing a more rigid multivariate testing. Of note, this result shows that high-level amplifications of individual genes, in contrast to low-level gains, contribute to a distinct clinical behaviour and therefore both of these parameters might be considered separately in this type of analysis.

The molecular mechanisms which promote tumour progression by CCNL1 remain to be determined. Up to now, only little is known about its function. CCNL1, like the closely related protein CCNL2, contain a N-terminal cyclin box but differs from the other members of the cyclin family by the presence of a so-called RSdomain, which is a hallmark of proteins involved in pre-mRNA processing (de Graaf et al, 2004; Yang et al, 2004). Both proteins were recently found to be colocalised with the splicing factor SC35 within nuclear speckles. CCNL1 was also identified as an immediate early gene after induction of several growth factors including epidermal growth factor (Berke et al, 2001), and there is evidence that it is involved in G0 to G1 cell cycle progression (Iyer et al, 1999). Recently, CCNL1 has been detected within a small amplicon on chromosome 3q25.3 in the HNSCC cell line CAL 27. Subsequent expression analyses revealed that it is recurrently overexpressed in primary HNSCC (Redon et al, 2002). Our data underline the pathogenic role of CCNL1 in HNSCC progression, since an increased copy number of this gene was associated with the formation of loco-regional metastases and an unfavourable clinical outcome.

\section{ACKNOWLEDGEMENTS}

We are indebted to Frauke Devens and Laura Pucchio for technical support. This work was supported by the Deutsche Krebshilfe (No. 10-1745-Ho), and the National Genome Research Network, Germany (No. KB-P2T02).

\section{REFERENCES}

Baatenburg de Jong RJ, Knegt P, Verwoerd CD (1993) Reduction of the number of neck treatments in patients with head and neck cancer. Cancer 71: 2312-2318

Berke JD, Sgambato V, Zhu PP, Lavoie B, Vincent M, Krause M, Hyman SE (2001) Dopamine and glutamate induce distinct striatal splice forms of Ania-6, an RNA polymerase II-associated cyclin. Neuron 32: 277-287

Bockmühl U, Schluns K, Kuchler I, Petersen S, Petersen I (2000) Genetic imbalances with impact on survival in head and neck cancer patients. Am J Pathol 157: 369-375

de Graaf K, Hekerman P, Spelten O, Herrmann A, Packman LC, Bussow K, Muller-Newen G, Becker W (2004) Characterization of cyclin L2, a novel cyclin with an arginine/serine-rich domain: phosphorylation by DYRK1A and colocalization with splicing factors. J Biol Chem 279: 4612-4624

Freier K, Joos S, Flechtenmacher C, Devens F, Benner A, Bosch FX, Lichter P, Hofele C (2003) Tissue microarray analysis reveals site-specific

prevalence of oncogene amplifications in head and neck squamous cell carcinoma. Cancer Res 63: 1179-1182

Guhaniyogi J, Brewer G (2001) Regulation of mRNA stability in mammalian cells. Gene 265: $11-23$

Hibi K, Trink B, Patturajan M, Westra WH, Caballero OL, Hill DE, Ratovitski EA, Jen J, Sidransky D (2000) AIS is an oncogene amplified in squamous cell carcinoma. Proc Natl Acad Sci USA 97: 5462-5467

Imoto I, Pimkhaokham A, Fukuda Y, Yang ZQ, Shimada Y, Nomura N, Hirai H, Imamura M, Inazawa J (2001) SNO is a probable target for gene amplification at 3q26 in squamous-cell carcinomas of the esophagus. Biochem Biophys Res Commun 286: 559-565

Iyer VR, Eisen MB, Ross DT, Schuler G, Moore T, Lee JC, Trent JM, Staudt LM, Hudson Jr J, Boguski MS, Lashkari D, Shalon D, Botstein D, Brown PO (1999) The transcriptional program in the response of human fibroblasts to serum. Science 283: $83-87$ 
Landis SH, Murray T, Bolden S, Wingo PA (1999) Cancer statistics, 1999. CA Cancer J Clin 49: 8-31

Redon R, Hussenet T, Bour G, Caulee K, Jost B, Muller D, Abecassis J, du Manoir S (2002) Amplicon mapping and transcriptional analysis pinpoint cyclin $\mathrm{L}$ as a candidate oncogene in head and neck cancer. Cancer Res 62: 6211-6217

Speicher MR, Howe C, Crotty P, du Manoir S, Costa J, Ward DC (1995) Comparative genomic hybridization detects novel deletions and amplifications in head and neck squamous cell carcinomas. Cancer Res 55: $1010-1013$

Stroschein SL, Wang W, Zhou S, Zhou Q, Luo K (1999) Negative feedback regulation of TGF-beta signaling by the SnoN oncoprotein. Science 286: $771-774$
Weber RG, Scheer M, Born IA, Joos S, Cobbers JM, Hofele C, Reifenberger G, Zoller JE, Lichter P (1998) Recurrent chromosomal imbalances detected in biopsy material from oral premalignant and malignant lesions by combined tissue microdissection, universal DNA amplification, and comparative genomic hybridization. Am J Pathol 153: 295-303

Yang A, Kaghad M, Wang Y, Gillett E, Fleming MD, Dotsch V, Andrews NC, Caput D, McKeon F (1998) TP73L, a p53 homolog at 3q27-29, encodes multiple products with transactivating, death-inducing, and dominantnegative activities. Mol Cell 2: 305-316

Yang L, Li N, Wang C, Yu Y, Yuan L, Zhang M, Cao X (2004) Cyclin L2, a novel RNA polymerase II-associated cyclin, is involved in pre-mRNA splicing and induces apoptosis of human hepatocellular carcinoma cells. J Biol Chem 279: 11639-11648 